\title{
A New Flexible Version of the Lomax Distribution with Applications
}

\author{
T. H. M. Abouelmagd ${ }^{1,2}$ \\ ${ }^{1}$ Management Information System Department, Taibah, University, Saudi Arabia. \\ ${ }^{2}$ Department of Statistics, Mathematics \& Insurance, Benha University, Egypt. \\ Correspondence: T. H. M. Abouelmagd. E-mail: tabouelmagd@taibahu.edu.sa
}

Received: June 26, 2018 Accepted: July 10, 2018 Online Published: August 27, 2018

doi:10.5539/ijsp.v7n5p120

URL: https://doi.org/10.5539/ijsp.v7n5p120

\begin{abstract}
A new version of the Lomax model is introduced and studied. The major justification for the practicality of the new model is based on the wider use of the Lomax model. We are also motivated to introduce the new model since the density of the new distribution exhibits various important shapes such as the unimodal, the right skewed and the left skewed. The new model can be viewed as a mixture of the exponentiated Lomax distribution. It can also be considered as a suitable model for fitting the symmetric, left skewed, right skewed, and unimodal data sets. The maximum likelihood estimation method is used to estimate the model parameters. We prove empirically the importance and flexibility of the new model in modeling two types of aircraft windshield lifetime data sets. The proposed lifetime model is much better than gamma Lomax, exponentiated Lomax, Lomax and beta Lomax models so the new distribution is a good alternative to these models in modeling aircraft windshield data.
\end{abstract}

Keywords: Lomax model, Order Statistics, Maximum Likelihood Estimation, Quantile function, Generating Function, Moments

\section{Introduction}

A random variable (rv) $W$ has the Lomax (Lx) distribution with two parameters $\lambda$ and $\beta$ if it has cumulative distribution function $(\mathrm{CDF})$ (for $w>0$ ) given by

$$
G_{\lambda, \beta}(w)=1-\left(1+w \beta^{-1}\right)^{-\lambda}
$$

where $\lambda>0$ and $\beta>0$ are the shape and scale parameters, respectively. Then the corresponding PDF of (1) is

$$
g_{\lambda, \beta}(w)=\lambda \beta^{-1}\left(1+w \beta^{-1}\right)^{-(\lambda+1)} .
$$

In the literature, the Lomax (Lx) or Pareto type II (PaII) model (see Lomax (1954)) was originally pioneered for modeling business failure data. The Lx distribution has found a wide application in many fields such as biological sciences, ctuarial science, engineering, size of cities, income and wealth inequality, amedical and reliability modeling. It has been applied to model data obtained from income and wealth (Harris (1968) and Atkinson and Harrison (1978)), firm size (Corbellini et al., (2007)), reliability and life testing (Hassan Al-Ghamdi (2009)), Hirschrelated statistics (Glanzel (2008)), for modeling gauge lengths data (Afify et al., (2015)), for modeling bladder cancer patients data and remission times data (Yousof et al., (2016) and Yousof et al., (2018)). According to Yousof et al. (2016) the CDF of the Burr X generator (BrX-G) is

$$
F_{\theta, \xi}(x)=2 \theta \int_{0}^{\frac{G(x ; \xi)}{\bar{G}(x ;)}} t\left[1-\exp \left(-t^{2}\right)\right]^{\theta-1} \exp \left(-t^{2}\right) d t=\left\{-\exp \left[-\left(\frac{G(x ; \xi)}{\bar{G}(x ; \xi)}\right)^{2}\right]+1\right\}^{\theta} .
$$

The PDF of the BrX-G is given by

$$
f_{\theta, \xi}(x)=2 \theta g(x ; \xi) \bar{G}(x ; \xi)^{-3} G(x ; \xi) \exp \left[-\left(\frac{G(x ; \xi)}{\bar{G}(x ; \xi)}\right)^{2}\right]\left\{-\exp \left[-\left(\frac{G(x ; \xi)}{\bar{G}(x ; \xi)}\right)^{2}\right]+1\right\}^{\theta-1},
$$

where $\theta$ is the shape parameter, $g(x ; \xi)$ and $G(x ; \xi)$ denote the PDF and CDF of the baseline model with parameter vector $\xi$ and $1-G(x ; \xi)=\bar{G}(x ; \xi)$. Inserting (1) in to (3) we get the the CDF of the Burr X Lomax (BrXLx) as

$$
F_{\theta, \lambda, \beta}(x)=\left(1-\exp \left\{-\left[\frac{1-\left(1+x \beta^{-1}\right)^{-\lambda}}{\left(1+x \beta^{-1}\right)^{-\lambda}}\right]^{2}\right)\right)^{\theta} \text {. }
$$

The PDF of the BrXLx is given by 


$$
\begin{aligned}
f_{\theta, \lambda, \beta}(x)= & 2 \theta \lambda \beta^{-1}\left(1+x \beta^{-1}\right)^{2 \lambda-1}\left[1-\left(1+x \beta^{-1}\right)^{-\lambda}\right] \\
& \times \exp \left\{-\left[\frac{1-\left(1+x \beta^{-1}\right)^{-\lambda}}{\left(1+x \beta^{-1}\right)^{-\lambda}}\right]^{2}\right\}\left(1-\exp \left\{-\left[\frac{1-\left(1+x \beta^{-1}\right)^{-\lambda}}{\left(1+x \beta^{-1}\right)^{-\lambda}}\right]\right\}\right)^{\theta-1} .
\end{aligned}
$$

We are motivated to introduce the new model since the PDF of the new distribution exhibits various important shapes such as the unimodal, the right skewed and the left skewed (see figurue 1). The new model can be viewed as a mixture of the exponentiated Lx distribution (see Subsection 2.1). It can also be considered as a suitable model for fitting the symmetric, left skewed, right skewed, and unimodal data sets (see aplications Section). The maximum likelihood estimation method is used to estimate the model parameters. We prove empirically the importance and flexibility of the new model in modeling two types of aircraft windshield lifetime data sets. The proposed lifetime model is much better than gamma Lx, beta Lx, exponentiated Lx and Lx models so the new model is a good alternative to these models in modeling aircraft windshield data.
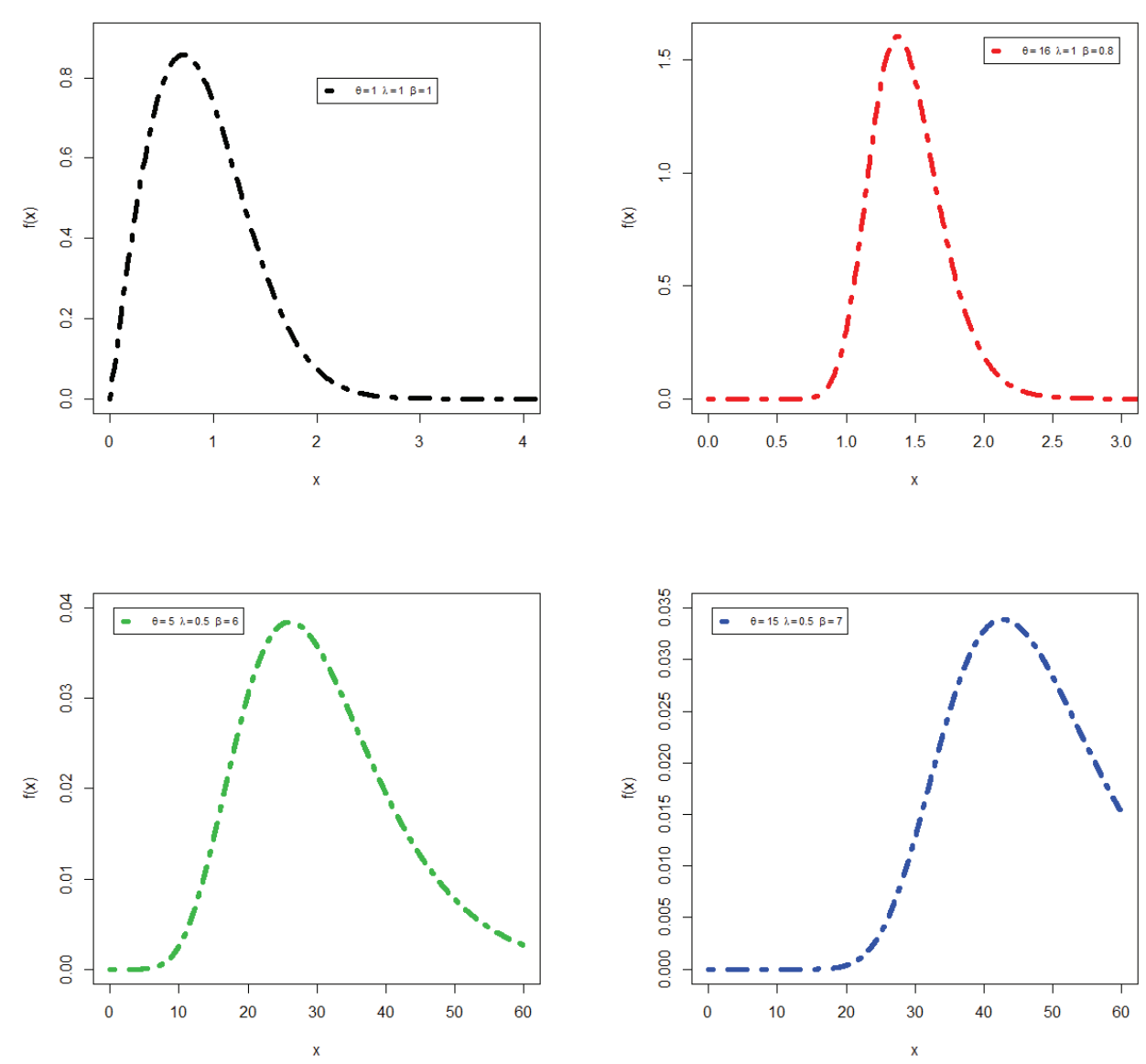

Figure 1. Plots of the BrXLx PDF 

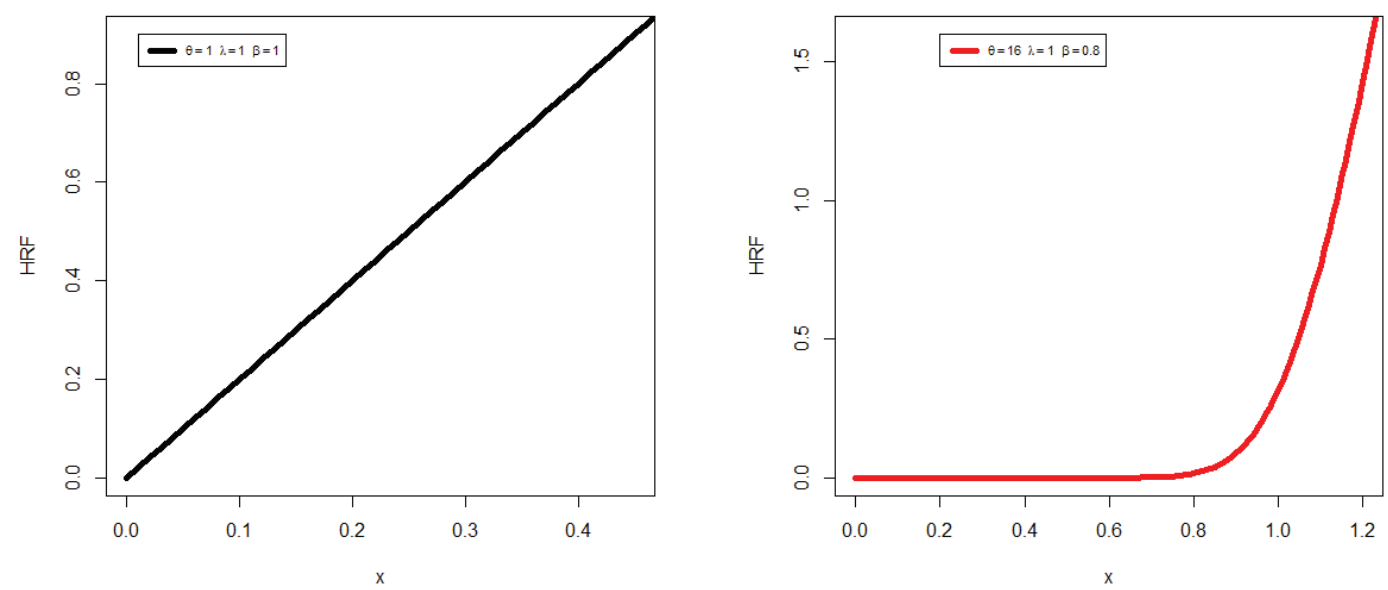

Figure 2. Plots of the BrXLx HRF

The reliability function (RF) $\left(R_{\theta, \lambda, \beta}(x)\right)$, hazard rate function (HRF) $\left(h_{\theta, \lambda, \beta}(x)\right)$, reversed hazard rate function (RHRF) $\left(r_{\theta, \lambda, \beta}(x)\right)$ and cumulative hazard rate function $(\mathrm{CHRF})\left(H_{\theta, \lambda, \beta}(x)\right)$ of $X$ are given, respectively, by

$$
\begin{aligned}
& R_{\theta, \lambda, \beta}(x)=1-\left(1-\exp \left\{-\left[\frac{1-\left(1+x \beta^{-1}\right)^{-\lambda}}{\left(1+x \beta^{-1}\right)^{-\lambda}}\right]^{2}\right\}\right)^{\theta}, \\
h_{\theta, \lambda, \beta}(x)= & 2 \theta \lambda \beta^{-1}\left(1+x \beta^{-1}\right)^{2 \lambda-1}\left[1-\left(1+x \beta^{-1}\right)^{-\lambda}\right] \\
& \left.\times 1-\left(1-\exp \left\{-\left[\frac{1-\left(1+x \beta^{-1}\right)^{-\lambda}}{\left(1+x \beta^{-1}\right)^{-\lambda}}\right]\right)^{\theta}\right]^{-1}\right]^{-\lambda} \\
& \times \exp \left\{-\left[\frac{1-\left(1+x \beta^{-1}\right)^{-\lambda}}{\left(1+x \beta^{-1}\right)^{-\lambda}}\right]^{2}\right\}\left(1-\exp \left\{-\left[\frac{1-\left(1+x \beta^{-1}\right)^{-\lambda}}{\left(1+x \beta^{-1}\right)^{-\lambda}}\right]^{2}\right)\right)^{\theta-1}, \\
r_{\theta, \lambda, \beta}(x)= & 2 \theta \lambda \beta^{-1}\left(1+x \beta^{-1}\right)^{2 \lambda-1}\left[1-\left(1+x \beta^{-1}\right)^{-\lambda}\right] \\
& \times \exp \left\{-\left[\frac{1-\left(1+x \beta^{-1}\right)^{-\lambda}}{\left(1+x \beta^{-1}\right)^{-\lambda}}\right]^{2}\right\}\left(1-\exp \left\{-\left[\frac{1-\left(1+x \beta^{-1}\right)^{-\lambda}}{\left(1+x \beta^{-1}\right)^{-\lambda}}\right]^{2}\right)\right)^{-1}
\end{aligned}
$$

and

$$
\left.H_{\theta, \lambda, \beta}(x)=-\left\{\log \left[1-\left(1-\exp \left\{-\left[\frac{1-\left(1+x \beta^{-1}\right)^{-\lambda}}{\left(1+x \beta^{-1}\right)^{-\lambda}}\right]^{2}\right\}\right)\right)^{\theta}\right]\right\}
$$




\section{Mathematical and Statistical Properties}

\subsection{Linear Representation}

In this section, we provide a very useful linear representation for the BX-G density function. If $|s|<1$ and $b>0$ is a real non-integer, the power series holds

$$
(1-s)^{a-1}=\sum_{h=0}^{\infty}\left\{(-1)^{h} \Gamma(a) s^{h} /[h ! \Gamma(a-h)]\right\} .
$$

Applying (7) to (6) we have

$$
f(x)=2 \theta \lambda \beta^{-1} \frac{\left[1-\left(1+x \beta^{-1}\right)^{-\lambda}\right]}{\left(1+x \beta^{-1}\right)^{1-2 \lambda}} \sum_{i=0}^{\infty} \frac{(-1)^{i} \Gamma(\theta)}{i ! \Gamma(\theta-i)} \exp \left\{-(1+i)\left[\frac{1-\left(1+x \beta^{-1}\right)^{-\lambda}}{\left(1+x \beta^{-1}\right)^{-\lambda}}\right]^{2}\right\} .
$$

Applying the power series to the term

$$
\exp \left\{-(1+i)\left[\frac{1-\left(1+x \beta^{-1}\right)^{-\lambda}}{\left(1+x \beta^{-1}\right)^{-\lambda}}\right]^{2}\right\}
$$

Equation (8) becomes

$$
f(x)=2 \theta \lambda \beta^{-1}\left(1+x \beta^{-1}\right)^{-(\lambda+1)} \sum_{i, j=0}^{\infty} \frac{(-1)^{i+j}(i+1)^{j} \Gamma(\theta)}{i ! j ! \Gamma(\theta-i)} \frac{\left[1-\left(1+x \beta^{-1}\right)^{-\lambda}\right]^{2 j+1}}{\left[\left(1+x \beta^{-1}\right)^{-\lambda}\right]^{2 j+3}} .
$$

Consider the series expansion

$$
(1-s)^{-a}=\left.\sum_{w=0}^{\infty}\left\{s^{w} \Gamma(a+w) /[w ! \Gamma(a)]\right\}\right|_{(|s|<1, a>0)} .
$$

Applying (10) to (9) for the term $\left[\left(1+x \beta^{-1}\right)^{-\lambda}\right]^{2 j+3}$ we get

$$
\begin{aligned}
f(x)= & 2 \theta \sum_{i, j, k=0}^{\infty} \frac{(-1)^{i+j}(i+1)^{j} \Gamma(\theta) \Gamma(2 j+k+3)[2 j+k+2]}{i ! j ! k ! \Gamma(\theta-i) \Gamma(2 j+3)[2 j+k+2]} \\
& \times \underbrace{\lambda \beta^{-1}\left(1+x \beta^{-1}\right)^{-(\lambda+1)}}_{g_{\lambda, \beta}(x)} \underbrace{\left[1-\left(1+x \beta^{-1}\right)^{-\lambda}\right]^{2 j+k+1}}_{G_{\lambda, \beta}(x)^{2 j+k+1}} .
\end{aligned}
$$

This can be written as

$$
f(x)=\sum_{j, k=0}^{\infty} \zeta_{j, k} \pi_{(2+2 j+k), \lambda, \beta}(x),
$$

where

$$
\zeta_{j, k}=\frac{2 \theta(-1)^{j} \Gamma(\theta) \Gamma(2 j+k+3)}{j ! k ! \Gamma(2 j+3)(2+2 j+k)} \sum_{i=0}^{\infty} \frac{(-1)^{i}(i+1)^{j}}{i ! \Gamma(\theta-i)}
$$

and

$$
\pi_{(2+2 j+k), \lambda,}(x)=(2+2 j+k) \underbrace{\left[1-\left(1+x \beta^{-1}\right)^{-\lambda}\right]^{2 j+k+1}}_{G_{\lambda, \beta}(x)^{2 j+k+1}} \underbrace{\lambda \beta^{-1}\left(1+x \beta^{-1}\right)^{-(\lambda+1)}}_{g_{\lambda, \beta}(x)} .
$$

The CDF of the BrXLx , similarly, can also be expressed as a mixture of exp-Lx CDFs as

$$
F(x)=\sum_{j, k=0}^{\infty} \zeta_{j, k} \Pi_{(2+2 j+k), \lambda,}(x),
$$

where

$$
\Pi_{(2+2 j+k), \lambda \beta}(x)=\left[1-\left(1+x \beta^{-1}\right)^{-\lambda}\right]^{2 j+k+2}
$$

is the CDF of the exp-Lx model with power parameter $2 j+k+2$. 


\section{Moments and Generating Function}

The $r$-th ordinary moment of $X$ is given by

$$
\mu_{r}^{\prime}=\mathbf{E}\left(X^{r}\right)=\int_{-\infty}^{\infty} x^{r} f(x) d x
$$

Then we obtain

where $\mathbf{B}(\cdot, \cdot)$ is the complete beta function, setting $r=1$ in (13), we have the mean of $X$

$$
E(X)=\mu_{1}^{\prime}=\left.\sum_{j, k=0}^{\infty} \sum_{w=0}^{r} \zeta_{j, k}(2+2 j+k) \beta(-1)^{w}\left(\begin{array}{c}
1 \\
w
\end{array}\right) \mathbf{B}\left((2+2 j+k), 1+\frac{w-1}{\lambda}\right)\right|_{(\lambda>1)} .
$$

Setting $r=2.3$ and 4 in (13), we have the 2-nd, 3-rd and the 4-th moments about the origin which can be used to obtain the central moments.

Table 1. Mean, variance, skewness and kurtosis of the BrXLx distribution with $\lambda=\beta=0.5$ and different values of $\theta$

\begin{tabular}{ccccc}
\hline \hline$\theta$ & Mean $(X)$ & Variance $(X)$ & Skew $(X)$ & $\operatorname{Kur}(X)$ \\
\hline \hline 0.001 & 0.0031329 & 0.00401742 & $\mathbf{3 5 . 2 2 6 1 3}$ & $\mathbf{1 6 8 5 . 7 8 6}$ \\
0.01 & 0.03090983 & 0.03913622 & 11.13911 & 171.0975 \\
0.1 & 0.27350260 & 0.3092640 & 3.534371 & 19.81156 \\
1 & 1.3862270 & 0.9077153 & 1.310273 & 5.486999 \\
5 & 2.6036300 & 0.9669048 & 1.004489 & 4.735616 \\
10 & 3.1402080 & 0.9367001 & 0.9842919 & 4.730640 \\
20 & 3.6686360 & 0.9007004 & 0.9865868 & 4.770655 \\
50 & 4.3510240 & 0.8554498 & 1.002323 & 4.843780 \\
100 & 4.8552040 & 0.8256333 & 1.016365 & 4.899162 \\
200 & 5.3500750 & 0.7998305 & 1.029670 & 4.949684 \\
400 & 5.8367520 & 0.7776043 & 1.041495 & 4.994194 \\
800 & 6.3162710 & 0.7583990 & 1.051718 & 5.032806 \\
1000 & 6.4692710 & 0.7527710 & 1.054685 & 5.044076 \\
\hline \hline
\end{tabular}

We proved, numerically, that the BrXLx model provides better fits than other four competitive extensions of the Lx models (see Section 6) so the BrXLx model is a exemplary alternative to these mosels. The skewness $(\operatorname{Skew}(X))$ of the BrXLx distribution can range in the interval $(35.23,0.17)$, whereas the kurtosis $(\operatorname{Kur}(X))$ of the BrXLx distribution varies only in the interval $(1685.7,2.61)$ also the mean of $X$ (Mean $(X))$ increases as $\theta$ increases, the skewness is always positive (see Table 1 and 2).

Table 2. Mean, variance, skewness and kurtosis of the BrXLx distribution with $\lambda=\beta=3.5$ and different values of $\theta$

\begin{tabular}{ccccc}
\hline \hline$\theta$ & Mean $(X)$ & Variance $(X)$ & Skew $(X)$ & $\operatorname{Kur}(X)$ \\
\hline \hline 0.001 & 0.0019254 & 0.000972489 & 23.04304 & 633.3245 \\
0.01 & 0.0189181 & 0.009325541 & 7.190108 & 63.41473 \\
0.1 & 0.1612188 & 0.06369933 & 1.989041 & 6.713972 \\
1 & 0.6706015 & 0.08385418 & 0.2087107 & $\mathbf{2 . 6 1 8 2 9 0}$ \\
5 & 1.016599 & 0.03950313 & $\mathbf{0 . 1 7 4 3 2 3 8}$ & 3.001886 \\
10 & 1.128713 & 0.02890308 & 0.2642751 & 3.103622 \\
20 & 1.224394 & 0.02192567 & 0.3539608 & 3.207029 \\
50 & 1.332142 & 0.01602527 & 0.4566714 & 3.347865 \\
100 & 1.403011 & 0.01306238 & 0.520966 & 3.452703 \\
200 & 1.466852 & 0.01089229 & 0.5750721 & 3.552538 \\
400 & 1.525029 & 0.009253848 & 0.6207442 & 3.64536 \\
800 & 1.578552 & 0.007983825 & 0.6595533 & 3.730876 \\
1000 & 1.594923 & 0.007634884 & 0.6708122 & 3.756873 \\
\hline \hline
\end{tabular}


The moment generating function (MGF) $M_{X}(t)=\mathbf{E}\left(e^{t X}\right)$ of $X$. Clearly, the first one can be derived from equation (10) as

$$
M_{X}(t)=\left.\sum_{j, k=0}^{\infty} \sum_{w=0}^{r} \zeta_{j, k}\left[t^{r} / r !\right](2+2 j+k) \beta^{r}(-1)^{w}\left(\begin{array}{c}
r \\
w
\end{array}\right) \mathbf{B}\left((2+2 j+k), 1+\frac{w-r}{\lambda}\right)\right|_{(\lambda>r)},
$$

\section{Incomplete Moments and Mean Deviations}

The $s$-th incomplete moment, say $I_{s}(t)$, of $X$ can be expressed from (10) as $I_{s}(t)=\int_{-\infty}^{t} x^{s} f(x) d x$, we have

$$
I_{s}(t)=\left.\sum_{j, k=0}^{\infty} \sum_{w=0}^{s} \zeta_{j, k}(2+2 j+k) \beta^{s}(-1)^{w}\left(\begin{array}{c}
s \\
w
\end{array}\right) \mathbf{B}_{t}\left((2+2 j+k), 1+\frac{w-s}{\lambda}\right)\right|_{(\lambda>s) .}
$$

The mean deviations about the mean

$$
\mathbf{E}(|X-\mathbf{E}(X)|)=M D_{(\text {mean })}=-2 I_{1}(\mathbf{E}(X))+2 \mathbf{E}(X) F(\mathbf{E}(X))
$$

and about the median

$$
\mathbf{E}(|X-\operatorname{Median}(X)|)=M D_{(\text {median })}=-2 I_{1}(\operatorname{Median}(X))+\mathbf{E}(X)
$$

of $X, F(\mathbf{E}(X))$ is easily calculated from (5) and $I_{1}(t)$ is the first incomplete moment given by (14) with $s=1$. Now, we provide two ways to determine $M D_{(\text {mean })}$ and $M D_{(\text {median })}$. The $I_{1}(t)$ can be derived from (14) as

$$
I_{1}(t)=\left.\sum_{j, k=0}^{\infty} \sum_{w=0}^{s} \zeta_{j, k}(2+2 j+k) \beta(-1)^{w}\left(\begin{array}{l}
1 \\
w
\end{array}\right) \mathbf{B}_{t}\left((2+2 j+k), 1+\frac{w-1}{\lambda}\right)\right|_{(\lambda>1)} .
$$

\subsection{Probability Weighted Moments (PWM)}

The $(s, r)$-th PWM of $X$ following the BrXLx model, say $\rho_{s, r}$, is formally defined by

Using (5), (6) we can write

$$
\rho_{s, r}=\mathbf{E}\left\{X^{s} F_{\theta, \lambda, \beta}(x)^{r}\right\}=\int_{-\infty}^{\infty} x^{s} F_{\theta, \lambda, \beta}(x)^{r} f_{\theta, \lambda, \beta}(x) d x .
$$

$$
F_{\theta, \lambda, \beta}(x)^{r} f_{\theta, \lambda, \beta}(x)=\sum_{j, k=0}^{\infty} v_{j, k} \pi_{2 j+k+2}(x)
$$

where

$$
v_{j, k}=\frac{2 \theta(-1)^{j}}{(2+2 j+k) j ! k !}[\Gamma(2 j+k+3) / \Gamma(2 j+3)] \sum_{i=0}^{\infty}(-1)^{i}(i+1)^{j}\left(\begin{array}{c}
\theta(r+1)-1 \\
i
\end{array}\right) .
$$

Then, the $(s, r)-$ th PWM of $X$ can be expressed as

$$
\rho_{s, r}=\left.\sum_{j, k=0}^{\infty} \sum_{w=0}^{s} v_{j, k}(2+2 j+k) \beta^{s}(-1)^{w}\left(\begin{array}{c}
s \\
w
\end{array}\right) \mathbf{B}\left((2+2 j+k), 1+\frac{w-s}{\lambda}\right)\right|_{(\lambda>s) .}
$$

\subsection{Moments of the Reversed Residual Life}

The $n$-th moment of the reversed residual life, say

$$
\Upsilon_{n}(t)=\mathbf{E}\left[\left.(t-X)^{n}\right|_{(X \leq t, t>0, n=1,2, \ldots)}\right],
$$

uniquely determines $F_{\theta, \lambda, \beta}(x)$. We obtain

$$
\Upsilon_{n}(t)=\frac{\int_{0}^{t}(t-x)^{n} d F_{\theta, \lambda, \beta}(x)}{F_{\theta, \lambda, \beta}(x)} .
$$

Then, the $n$-th moment of the reversed residual life of $X$ becomes

$$
\Upsilon_{n}(t)=\left.F_{\theta, \lambda, \beta}(x)^{-1} \sum_{j, k=0}^{\infty} \sum_{r=0}^{n} \sum_{w=0}^{n} \zeta_{j, k, r, w} \beta^{n}\left(\begin{array}{l}
n \\
r
\end{array}\right)\left(\begin{array}{l}
n \\
w
\end{array}\right) \mathbf{B}_{t}\left((2+2 j+k), 1+\frac{w-n}{\lambda}\right)\right|_{(\lambda>n)} .
$$


where

$$
\zeta_{j, k, r, w}=\zeta_{j, k}(-1)^{r} t^{n-r}(-1)^{w}(2+2 j+k),
$$

and

$$
\mathbf{B}_{a_{3}}\left(a_{1} ; a_{2}\right)=\int_{0}^{a_{3}} z^{a_{1}-1}(1-z)^{a_{2}-1} d z=
$$

is the incomplete beta function.

\subsection{Reliability Estimation}

The most widely approach used for reliability estimation is the stress-strength model (SSM), this model is used in many applications of physics and engineering such as strength failure and system collapse. In SSM, we have

$$
\mathbf{R}\left(T_{1},\left.T_{2}\right|_{\left(T_{2}<T_{1}\right)}\right)=\operatorname{Pr}\left(T_{2}<T_{1}\right)
$$

is a measure of reliability of the system when it is subjected to random stress $T_{2}$ and has strength $T_{1}$. The system fails if and only if the applied stress is greater than its strength $\left(T_{1}<T_{2}\right)$. Other interpretation can be given as the reliability $\mathbf{R}\left(T_{1},\left.T_{2}\right|_{\left(T_{2}<T_{1}\right)}\right)$ of a system is the probability that the system is strong enough to overcome the stress imposed on it. Let $T_{1}$ and $T_{2}$ be two independent rvs with $\operatorname{BrXLx}\left(\theta_{1}, \lambda, \beta\right)$ and $\operatorname{BrXLx}\left(\theta_{2}, \lambda, \beta\right)$ distributions, respectively. The PDF of $T_{1}$ and the CDF of $T_{2}$ can be written from Equations (6) and (5), respectively as

$$
f_{\theta_{1}, \lambda, \beta}^{(1)}(t)=2 \theta_{1} \sum_{i, j, k=0}^{\infty} \frac{(-1)^{i+j}(i+1)^{j} \Gamma(2 j+k+3) \Gamma\left(\theta_{1}\right)}{i ! j ! k ! \Gamma(2 j+3) \Gamma\left(\theta_{1}-i\right)} \underbrace{\left[1-\left(1+t \beta^{-1}\right)^{-\lambda}\right]^{2 j+k+1}}_{G_{\lambda, \beta}(t)^{2 J+K+1}} \underbrace{\lambda \beta^{-1}\left(1+t \beta^{-1}\right)^{-(\lambda+1)}}_{g_{\lambda, \beta}(t)}
$$

and

$$
F_{\theta_{2}, \lambda, \beta}^{(2)}(t)=2 \theta_{2} \sum_{h, w, m=0}^{\infty} \frac{(-1)^{h+w}(h+1)^{w} \Gamma(2 w+m+3) \Gamma\left(\theta_{2}\right)}{h ! w ! m !(2 w+m+2) \Gamma(2 w+3) \Gamma\left(\theta_{2}-h\right)} \underbrace{\left[1-\left(1+t \beta^{-1}\right)^{-\lambda}\right]^{2 j+k+2}}_{G_{\lambda, \beta}(t)^{2 J+K+2}} .
$$

Then, the reliability is defined by

$$
\mathbf{R}\left(T_{1},\left.T_{2}\right|_{\left(T_{2}<T_{1}\right)}\right)=\int_{0}^{\infty} f_{\theta_{2}, \lambda, \beta}^{(1)}(x) F_{\theta_{2}, \lambda, \beta}^{(2)}(x) d x .
$$

We can write

$$
\mathbf{R}\left(T_{1},\left.T_{2}\right|_{\left(T_{2}<T_{1}\right)}\right)=\sum_{j, k, w, m=0}^{\infty} \Psi_{j, k, w, m} \int_{0}^{\infty} \pi_{4+2 j+2 w+k+m}(t) d t
$$

where

$$
r_{j, k, w, m}=4 \theta_{1} \theta_{2} \sum_{j, k, w, m=0}^{\infty} \frac{(-1)^{j+w} \Gamma(2 w+m+3) \Gamma(2 j+k+3)}{j ! k ! w ! m ! \Gamma\left(\theta_{2}-h\right) \Gamma(2 w+3) \Gamma(2 j+3)} \sum_{i, h=0}^{\infty} \frac{(-1)^{i+h}(h+1)^{w}(i+1)^{j}\left(\begin{array}{c}
\theta_{1}-1 \\
i
\end{array}\right)\left(\begin{array}{c}
\theta_{2}-1 \\
h
\end{array}\right)}{(2 j+k+2 w+m+4)(2 w+m+2)}
$$

and

$$
\pi_{4+2 j+2 w+k+m}(t)=(2 j+k+2 w+m+4) \underbrace{\lambda \beta^{-1}\left(1+t \beta^{-1}\right)^{-(\lambda+1)}}_{g_{\lambda, \beta}(x)} \underbrace{\left[1-\left(1+t \beta^{-1}\right)^{-\lambda}\right]^{2 j+k+2 w+m+3}}_{G_{\lambda, \beta}(x)^{2 j+k+2 w+m+3}} .
$$

Thus, the reliability, $\mathbf{R}\left(T_{1},\left.T_{2}\right|_{\left(T_{2}<T_{1}\right)}\right)$, can be expressed as

$$
\mathbf{R}\left(T_{1},\left.T_{2}\right|_{\left(T_{2}<T_{1}\right)}\right)=\sum_{j, k, w, m=0}^{\infty} r_{j, k, w, m} .
$$




\subsection{Order Statistics}

Let $X_{1}, \ldots, X_{n}$ be a random sample (RS) from the BrXLx distribution and let $X_{1: n}, \ldots, X_{n: n}$ be the corresponding order statistics. The PDF of $i$-th order statistic, say $X_{i: n}$, can be expressed as

$$
f_{\theta, \lambda, \beta}^{(i: n)}(x)=\mathbf{B}^{-1}(i, n-i+1) f_{\theta, \lambda, \beta}(x) \sum_{j=0}^{n-i}(-1)^{j}\left(\begin{array}{c}
n-i \\
j
\end{array}\right) F_{\theta, \lambda, \beta}(x)^{j+i-1},
$$

using (5), (6) and (15) we get

$$
f_{\theta, \lambda, \beta}(x) F_{\theta, \lambda, \beta}(x)^{j+i-1}=\sum_{w, k=0}^{\infty} a_{w, k} \pi_{2 w+k+2}(x),
$$

where

$$
a_{w, k}=\frac{2 \theta(-1)^{w} \Gamma(2 w+k+3)}{w ! k ! \Gamma(2 w+3)(2 w+k+2)} \sum_{m=0}^{\infty}(-1)^{m}(m+1)^{w}\left(\begin{array}{c}
\theta(j+i)-1 \\
m
\end{array}\right) .
$$

The PDF of $X_{i: n}$ can be written as

$$
f_{\theta, \lambda, \beta}^{(i: n)}(x)=\sum_{w, k=0}^{\infty} \sum_{j=0}^{n-i}(-1)^{j}\left(\begin{array}{c}
n-i \\
j
\end{array}\right) a_{w, k} \pi_{2 w+k+2}(x) .
$$

Then, the density function of the BrXLx order statistics is a mixture of eponentiated Lomax (ELx). The $q-$ th moments of $X_{i: n}$ can be expressed as

$$
\mathbf{E}\left(X_{i: n}^{q}\right)=\left.\sum_{w, k=0}^{\infty} \sum_{j=0}^{n-i} \sum_{m=0}^{q} a_{w, k, j, m} \beta^{q}\left(\begin{array}{c}
q \\
m
\end{array}\right) \mathbf{B}\left((2 w+k+2), 1+\frac{m-q}{\lambda}\right)\right|_{(\lambda>q)} .
$$

where

$$
a_{w, k}(-1)^{j+m}(2+2 w+k) \mathbf{B}^{-1}(i, n-i+1)\left(\begin{array}{c}
n-i \\
j
\end{array}\right)\left(\begin{array}{c}
q \\
m
\end{array}\right)=a_{w, k, j, m}
$$

\subsection{Quantile Spread (QS) Ordering}

The QS of the $\operatorname{rv} U \sim \operatorname{BrXLx}(\theta, \lambda, \beta)$ having $\operatorname{CDF}(5)$ is given by

$$
\left.Q S_{U}(\xi)\right|_{(\xi \in(0.5,1))}=-F^{-1}(1-\xi)+F^{-1}(\xi)
$$

which implies

$$
Q S_{U}(\xi)=-\left[S^{-1}(\xi)\right]+\left[S^{-1}(1-\xi)\right]
$$

where

$$
S(u)=1-F(u) \text { and } F^{-1}(\xi)=S^{-1}(1-\xi)
$$

is the survival function. The QS of a any distribution describes how the probability mass is placed symmetrically about its median and hence it can be used to formalize concepts such as peakedness and tail weight traditionally associated with the kurtosis. So, it allows use to separate concepts of the kurtosis and peakedness for asymmetric models. Let $U_{1}$ and $U_{2}$ be two rvs following the BrXLx model with $Q S_{U_{1}}$ and $Q S_{U_{2}}$. Then $U_{1}$ is called smaller than $U_{2}$ in quantile spread order, denoted as $U_{1} \leq\{Q S\} U_{2}$, if

$$
\left.Q S_{U_{1}}(\xi)\right|_{(\xi \in(0.5,1))} \leq Q S_{U_{2}}(\xi) .
$$

Following are some properties of the QS order which can be obtained.

The order $\leq_{\{Q S\}}$ is a location-free

$$
U_{1} \leq_{\{Q S\}} U_{2} \text { if }\left(U_{1}+a\right) \leq\left._{\{Q S\}} U_{2}\right|_{(a \in \mathbb{R})}
$$

The order $\leq_{\{Q S\}}$ is dilative

$$
U_{1} \leq_{\{Q S\}} a U_{1} \text { whenever } a \geq 1 \text { and } U_{2} \leq\left._{\{Q S\}} a U_{2}\right|_{(\mathbf{C} \geq 1)} .
$$

Let $F_{U_{1}}$ and $F_{U_{2}}$ be symmetric, then

$$
U_{1} \leq_{\{Q S\}} U_{2} \text { if, and only if } F_{U_{1}}^{-1}(\xi) \leq\left. F_{U_{2}}^{-1}(\xi)\right|_{(\xi \in(0.5,1))} .
$$


The order $\leq_{\{Q S\}}$ implies ordering of the mean absolute deviation around the median, say $\left.\pi\left(U_{i}\right)\right|_{(i=1,2)}$,

$$
\mathbf{E}\left[\left|U_{1}-\operatorname{Median}\left(U_{1}\right)\right|\right]=\pi\left(U_{1}\right)
$$

and

$$
\mathbf{E}\left[\left|U_{2}-\operatorname{Median}\left(U_{2}\right)\right|\right]=\pi\left(U_{2}\right),
$$

where

$$
\pi\left(U_{1}\right) \leq_{\{Q S\}} \pi\left(U_{2}\right) \Leftarrow U_{1} \leq_{\{Q S\}} U_{2},
$$

finally

$$
U_{1} \leq_{\{Q S\}} U_{2} \text { if, and only if }-U_{1} \leq_{\{Q S\}}-U_{2} \text {. }
$$

\subsection{Entropies}

The Rényi entropy is defined by

$$
\left.I_{\delta}(X)\right|_{(\delta>0 \text { and } \delta \neq 1)}=\frac{\log \int_{-\infty}^{\infty} f_{\theta, \lambda, \beta}(x)^{\delta} d x}{1-\delta} .
$$

Using PDF (4), we can write

$$
\left.f(x)^{\delta}\right|_{(\delta>0 \text { and } \delta \neq 1)}=\sum_{j, k=0}^{\infty} \tau_{j, k} G_{\lambda, \beta}(x)^{\delta+2 j+k} g_{\lambda, \beta}(x)^{\delta},
$$

where

$$
\tau_{j, k}=\sum_{i=0}^{\infty} \frac{2^{\delta} \theta^{\delta}\left[(\theta \delta-\delta)_{i}\right](-1)^{i+j} \Gamma(3 \delta+2 j+k)}{i ! j ! k ! \Gamma(3 \delta+2 j)(\delta+i)^{-j}} .
$$

Then, the Rényi entropy of the BrXLx is given by

$$
I_{\delta}(X)=\frac{1}{1-\delta} \log \left\{\sum_{j, k=0}^{\infty} \tau_{j, k} \int_{0}^{\infty} G_{\lambda, \beta}(x)^{\delta+2 j+k} g_{\lambda, \beta}(x)^{\delta} d x\right\},
$$

The $\delta$-entropy, say $E_{\delta}(X)$, can be obtained as

$$
E_{\delta}(X)=\frac{1}{\delta-1} \log \left\{1-\left[\sum_{j, k=0}^{\infty} \tau_{j, k} \int_{-\infty}^{\infty} G_{\lambda, \beta}(x)^{q+2 j+k} g_{\lambda, \beta}(x)^{q} d x\right]\right\} .
$$

The Shannon entropy of a rv $X$, say $S E$, is defined by

$$
S E=E\{-[\log f(X)]\},
$$

follows by taking the limit of $I_{\delta}(X)$ as $\delta$ tends to 1 .

\section{Parameter Estimation}

Let $x_{1}, \ldots, x_{n}$ be a RS from the BrXLx model with parameters $\theta$ and $\xi$. Let $\Theta=(\theta, \lambda, \beta)^{\top}$ be the $3 \times 1$ parameter vector. For determining the maximum likelihood estimation (MLE) of $\Theta$, we have the $\log$-likelihood ( $\log \mathrm{L}$ ) function

$$
\begin{aligned}
\ell= & \ell(\Theta)=n \log 2+n \log \theta+n \log \lambda-n \log \beta+(2 \lambda-1) \sum_{i=1}^{n} \log \left(1+x_{i} \beta^{-1}\right) \\
& -\sum_{i=1}^{n}\left[\frac{1-\left(1+x_{i} \beta^{-1}\right)^{-\lambda}}{\left(1+x_{i} \beta^{-1}\right)^{-\lambda}}\right]^{2}+(\theta-1) \sum_{i=1}^{n} \log \left(1-\exp \left\{-\left[\frac{1-\left(1+x_{i} \beta^{-1}\right)^{-\lambda}}{\left(1+x_{i} \beta^{-1}\right)^{-\lambda}}\right]^{2}\right)\right)
\end{aligned}
$$

The components of the score vector, $\mathbf{U}(\Theta)=\left(\frac{\partial}{\partial \theta} \ell(\Theta), \frac{\partial}{\partial \lambda} \ell(\Theta), \frac{\partial}{\partial \beta} \ell(\Theta)\right)^{\top}$, are availble if needed, Via setting the nonlinear system of equations $U_{\theta}=0, U_{\gamma}=0$ and $U_{\beta}=0$ and solving them simultaneously yields the MLE $\widehat{\Theta}=(\widehat{\theta}, \widehat{\lambda}, \widehat{\beta},)^{\top}$. To solve those equations, it is usually more convenient to use the nonlinear optimization methods such as the quasi-Newton algorithm to numerically maximize $\ell(\Theta)$. 


\section{Applications}

In this section, we provide two applications to two real data sets to prove the importance and flexibility of the BrXLx distribution. We compare the fit of the BrXLx with competitve models namely: ELx model (Gupta et al., 1998), gamma Lomax (GLx) model (Cordeiro et al., 2015), beta Lomax (BLX) model (Lemonte and Cordeiro, 2013) and Lx model. The $\mathrm{CDFs}$ of these distributions are, respectively, given by (for $x>0$ and $\alpha, \beta, \lambda, a>0$ ):

$$
\begin{aligned}
F_{\alpha, \beta, \lambda}(x) & =\left[1-\left(1+x \beta^{-1}\right)^{-\lambda}\right]^{\alpha}, \\
F_{\alpha, \beta, \lambda}(x) & =\Gamma\left(\alpha ; \lambda \log \left[1+x \beta^{-1}\right]\right) \Gamma^{-1}(\alpha), \text { and } \\
F_{\alpha, \beta, \theta, \lambda}(x) & =\frac{\mathbf{B}_{\left[1-\left(1+x \beta^{-1}\right)^{-\lambda}\right]}(\alpha, \theta)}{\mathbf{B}(\alpha, \theta)},
\end{aligned}
$$

where $\Gamma(\cdot ; \cdot)$ is the incomplete gamma function.

The first real data set represents the data on failure times of 84 aircraft windshield given in Murthy et al. (2004). The data are:

4.1671.281,3, 4.035, 2.3000, 3.344, 4.602, 1.7570, 2.324, 2.625, 3.5780, 0.943, 4.121, 1.3030, 2.089, 2.632, 2.135, 2.962, $2.688,2.902,0.557,1.9110,1.568,3.5950,1.0700,4.2550,1.8990,2.610,3.4780,1.248,2.0100,1.914,1.505,2.154$, $2.9640,4.278,1.506,0.309,1.2810,1.9120,3.9240,2.190,3.000,4.3050,3.3760,2.6460,3.699,1.4320,2.097,2.934$, $4.2400,1.480,2.1940,3.103,4.376,1.615,2.2230,0.0400,1.866,2.3850,3.443,0.3010,1.876,2.4810,3.467,4.663$, $2.0850,2.890,2.038,2.820,1.1240,1.981,2.661,3.77903 .114,4.449,1.6190,2.224,3.1170,4.485,1.652,2.2290$, $3.166,4.570,1.652$.

The second real data set (recently studied by Tahir et al. (2015)) represents the data on service times of 63 aircraft windshield given in Murthy et al. (2004). The data are:

0.046, 1.436, 1.0030, 2.137, 3.500, 1.0100, 2.141, 3.6220, 1.085, 2.163, 2.592, 0.140, 1.492, 2.600, 0.150, 1.580, 2.670, $0.248,1.7190,2.717,2.820,0.389,1.9200,0.3130,1.915,1.1520,2.2400,4.015,1.183,2.878,0.487,1.9630,2.950$, $0.622,1.978,3.0030,0.2800,1.794,2.819,2.053,3.1020,0.952,2.065,3.3040,0.9960,0.9000,1.092,2.183,3.695$, $2.117,3.483,3.6650,2.3410,4.628,1.2440,2.435,4.806,1.249,2.4640,4.881,1.262,2.5430,5.140$.

In order to make a real comparison among the distributions, the estimated Log L values $(\widehat{\ell})$, Akaike Information Criteria (AIC), Cramer von Mises $\left(W^{*}\right)$ and Anderson-Darling $\left(A^{*}\right)$ goodness of-fit statistics were calculated for all competitive models. The statistics $W^{*}$ and $A^{*}$ were defined in Chen and Balakrishnan (1995) with details. In general, it can be chosen as the best model which has the smaller values of the $W^{*}, A^{*}$ and AIC statistics and the larger values of $(\widehat{\ell})$. The below computations are obtained via the "maxLik" and "goftest" sub-routines using the R-software. The analysis results are listed in Tables 3, 4, 5 and 6 . These results show obviously that the new distribution has the lowest $W^{*}, A I C$ and $A^{*}$ values and has the biggest estimated $-\widehat{\ell}$ among all the fitted models. Hence, it could be chosen as the best model under these criteria. From tables 3 and 5, the new model is much better than all competitive (BLx, ELx, GLx and Lx) models so the new model is a adequate alternative to these models in modeling aircraft windshield data.

Table 3. MLEs, standard erros of the estimates (in parentheses) for the first data set

\begin{tabular}{ccccc}
\hline \hline Model & $\widehat{\alpha}$ & $\widehat{\beta}$ & $\widehat{\theta}$ & $\widehat{\lambda}$ \\
\hline \hline BrXLx & & $5.237231 \times \mathrm{e}^{5}$ & $8.229086 \times \mathrm{e}^{-1}$ & $1.159193 \times \mathrm{e}^{5}$ \\
& & $(0.000)$ & $(0.1051083)$ & $(5446.8)$ \\
BLx & 3.6036 & 118.8374 & 33.6387 & 4.8307 \\
& $(0.6187)$ & $(63.7145)$ & $(9.2382)$ & $(429.0000)$ \\
ELx & 3.6261 & 26257.6808 & & 20074.5097 \\
& $(0.6236)$ & $(99.7417)$ & & $(2041.8263)$ \\
GLx & 3.5876 & 37029 & & 52001 \\
& $(0.5133)$ & $(81.1644)$ & & $(7955)$ \\
Lx & & 131789 & & 51425 \\
& & $(296.1200)$ & & $(5933.49)$ \\
\hline \hline
\end{tabular}


Table 4. $-\hat{\ell}$ and goodness-of-fits statistics for the first data set

\begin{tabular}{ccccc}
\hline \hline Model & $-\hat{\ell}$ & $A I C$ & $W^{*}$ & $A^{*}$ \\
\hline \hline BrXLx & $\mathbf{1 2 7 . 6 6 5}$ & $\mathbf{2 6 1 . 3 2 9 4}$ & $\mathbf{0 . 0 7 6 4 1 6 4}$ & $\mathbf{0 . 5 8 4 4 3 8}$ \\
BLx & 138.7177 & 285.4354 & 1.4084 & 0.1680 \\
ELx & 141.3997 & 288.7994 & 1.7435 & 0.2194 \\
GLx & 138.4042 & 282.8093 & 1.3667 & 0.1619 \\
Lx & 164.9900 & 333.9767 & 1.3976 & 0.1665 \\
\hline \hline
\end{tabular}
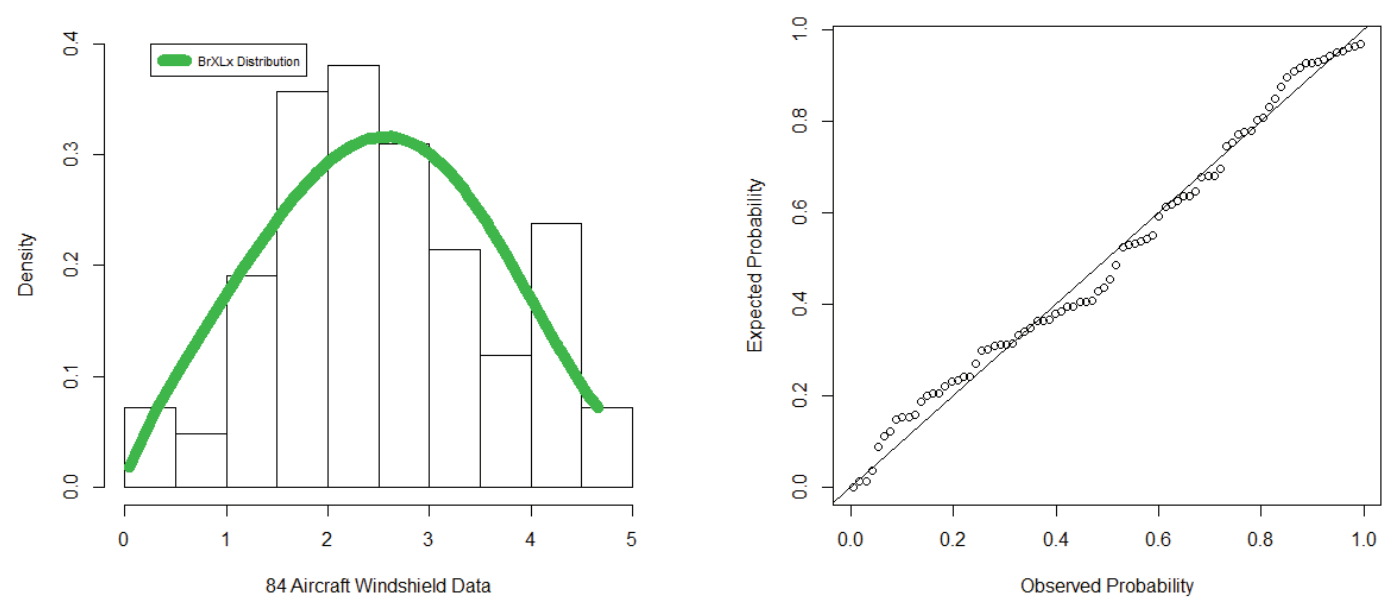

Figure 3. The fitted PDF and PP plot for the first data set

Table 5. MLEs, standard erros of the estimates (in parentheses) for the second data set

\begin{tabular}{ccccc}
\hline \hline Model & $\widehat{\alpha}$ & $\widehat{\beta}$ & $\widehat{\theta}$ & $\widehat{\lambda}$ \\
\hline \hline BrXLx & & 0.6467194 & 0.5987192 & 1.6211236 \\
& & $(0.0474756)$ & $(0.3901375)$ & $(0.9591999)$ \\
BLx & 1.9218 & 169.5800 & 31.2595 & 4.9685 \\
& $(0.3185)$ & $(339.2068)$ & $(316.8413)$ & $(50.5279)$ \\
ELx & 1.9145 & 32881.9 & & 22971.2 \\
& $(0.3483)$ & $(162.2230)$ & & $(3209.5)$ \\
GLx & 1.9073 & 39197.6 & & 35842.4 \\
& $(0.3214)$ & $(151.6530)$ & & $(6945)$ \\
Lx & & 207019 & & 99269 \\
& & $(301.2370)$ & & $(11863.5222)$ \\
\hline \hline
\end{tabular}



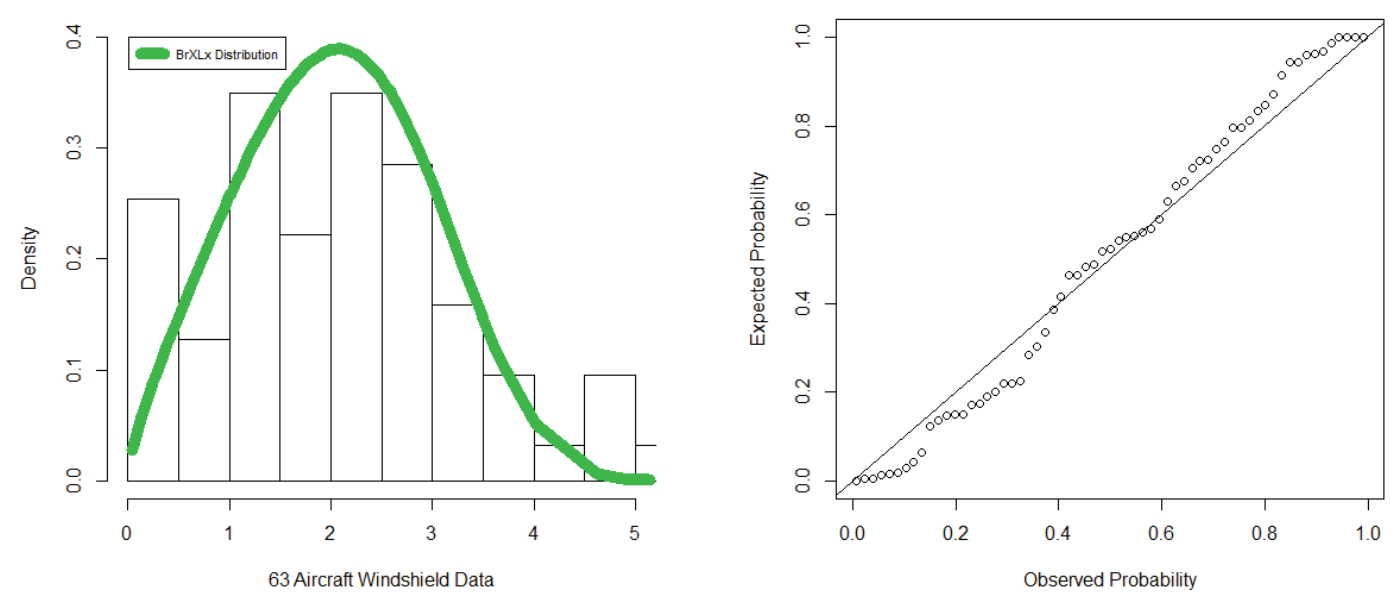

Figure 4. The fitted PDF and PP plot for the second data set

Table 6. $-\hat{\ell}$ and goodness-of-fits statistics for the second data set

\begin{tabular}{ccccc}
\hline \hline Model & $-\hat{\ell}$ & $A I C$ & $W^{*}$ & $A^{*}$ \\
\hline \hline BrXLx & $\mathbf{9 8 . 1 0 2 9 4}$ & $\mathbf{2 0 2 . 2 0 6}$ & $\mathbf{0 . 0 8 7 6 3}$ & $\mathbf{0 . 5 2 7 7 8 4}$ \\
BLx & 102.9611 & 213.9223 & 1.1336 & 0.1872 \\
ELx & 103.5468 & 213.9223 & 1.2331 & 0.2037 \\
GLx & 102.8333 & 211.6664 & 1.1121 & 0.2038 \\
Lx & 109.2988 & 222.5976 & 1.1265 & 0.1861 \\
\hline \hline
\end{tabular}

\section{Conclusions}

In this work, a new lifetime model called the Burr X Lomax (BrXLx) is introduced and studied. The major justification for introducing and studying the BrXLx model is based on the wider use of the Lx model in applied fields. We are also motivated to introduce and study the BrXLx model since the density of the BrXLx distribution displays various important shapes such as the unimodal, the right skewed and the left skewed. The new model can be viewed as a mixture of the exponentiated Lx distribution. It can also be considered as a convenient model for fitting the symmetric, the left skewed, the right skewed, and the unimodal data sets. The maximum likelihood estimation method is used to estimate the BrXLx parameters. We prove empirically the importance and flexibility of the BrXLx in modeling two types of aircraft windshield lifetime data. The proposed BrXLx lifetime model is much better than gamma Lomax, beta Lomax, exponentiated Lomax and Lomax models so the exponentiated Lomax, model is a good alternative to these models in modeling aircraft windshield data.

\section{References}

Afify, A. Z., Nofal, Z. M., Yousof, H. M., El Gebaly, Y. M., \& Butt, N. S. (2015). The transmuted Weibull Lomax distribution: properties and application. Pak. J. Stat. Oper. Res., 11(1), 135-152.

Atkinson, A. B., \& Harrison, A. J. (1978). Distribution of Personal Wealth in Britain (Cambridge University Press, Cambridge).

Chen, G., \& Balakrishnan, N. (1995). A general purpose approximate goodness-of-fit test. Journal of Quality Technology, 27, 154-161.

Gupta, R. C., Gupta, P. L., \& Gupta, R. D. (1998). Modeling failure time data by Lehman alternatives. Communications in Statistics-Theory \& methods, 27(4), 887-904.

Corbellini, A., Crosato, L., Ganugi, P., \& Mazzoli, M. (2007). Fitting Pareto II distributions on firm size: Statistical 
methodology and economic puzzles. Paper presented at the International Conference on Applied Stochastic Models and Data Analysis, Chania, Crete.

Cordeiro, G. M., Ortega, E. M., \& Popovic, B. V. (2015). The gamma-Lomax distribution. Journal of Statistical computation \& Simulation, 85(2), 305-319.

Glanzel, W. (2008). On some new bibliometric applications of statistics related to the h-index. Scientometrics, 77, 187196.

Hamedani, G. G. Rasekhi, M., Najibi, S. M., Yousof, H. M., \& Alizadeh, M. (2018). Type II general exponential class of distributions. Pak. J. Stat. Oper. Res., forthcoming.

Harris, C. M. (1968). The Pareto distribution as a queue service descipline. Operations Research, 16, 307-313.

Hassan, A. S., \& Al-Ghamdi, A. S. (2009). Optimum step stress accelerated life testing for Lomax distibution. Journal of Applied Sciences Research, 5, 2153-2164.

Lemonte, A. J., \& Cordeiro, G. M. (2013). An extended Lomax distribution. Statistics, 47(4), 800-816.

Lomax, K. S. (1954). Business failures: Another example of the analysis of failure data. Journal of the American Statistical Association, 49, 847-852.

Murthy, D. N. P., Xie, M., \& Jiang, R. (2004). Weibull Models, Wiley.

Silva, F. S., Percontini, A., de Brito, E., Ramos, M. W., Venancio, R., \& Cordeiro, G. M. (2017). The Odd Lindley-G Family of Distributions. Austrian Journal of Statistics, 46(1), 65-87.

Tahir, M. H., Cordeiro, G. M., Mansoor, M., \& Zubair, M. (2015). The Weibull-Lomax distribution: properties and applications. Hacettepe Journal of Mathematics \& Statistics, 44(2), 461-480.

Yousof, H. M., Afify, A. Z., Hamedani, G. G., \& Aryal, G. (2017). The Burr X generator of distributions for lifetime data. Journal of Statistical Theory \& Applications, 16, 288-305.

Yousof, H. M., Rasekhi, M., Alizadeh, M., \& Hamedani G. G. (2018). The Marshall-Olkin exponentiated generalized G family of distributions: properties, applications and characterizations. Proyecciones Journal of Mathematics, forthcoming.

\section{Copyrights}

Copyright for this article is retained by the author(s), with first publication rights granted to the journal.

This is an open-access article distributed under the terms and conditions of the Creative Commons Attribution license (http://creativecommons.org/licenses/by/4.0/). 